\title{
Traffic-related air pollution exposure over a 5-year period is associated with increased risk of asthma and poor lung function in middle age
}

\author{
Gayan Bowatte (1) ${ }^{1}$, Bircan Erbas², Caroline J. Lodge ${ }^{1,3}$, Luke D. Knibbs ${ }^{4}$, \\ Lyle C. Gurrin ${ }^{1}$, Guy B. Marks ${ }^{5,6}$, Paul S. Thomas ${ }^{7}$, David P. Johns ${ }^{8}$, \\ Graham G. Giles ${ }^{1,9,10}$, Jennie Hui ${ }^{11,12,13,14}$, Martine Dennekamp ${ }^{10,15}$ \\ Jennifer L. Perret ${ }^{1}$, Michael J. Abramson ${ }^{10}$, E. Haydn Walters ${ }^{8}$, \\ Melanie C. Matheson $\mathbb{1}^{1,16}$ and Shyamali C. Dharmage ${ }^{1,3,16}$
}

@ERSpublications

TRAP is associated with increased risks of asthma and reduced lung function in middle-aged adults http://ow.ly/Yt2n30etMkS

Cite this article as: Bowatte G, Erbas B, Lodge CJ, et al. Traffic-related air pollution exposure over a 5-year period is associated with increased risk of asthma and poor lung function in middle age. Eur Respir J 2017; 50: 1602357 [https://doi.org/10.1183/13993003.02357-2016].

ABSTRACT Current evidence concerning the impact of exposure to traffic-related air pollution (TRAP) on adult respiratory morbidity mainly comes from cross-sectional studies. We sought to establish more robust measures of this association and potential gene-environment interactions using longitudinal data from an established cohort study.

Associations between measures of TRAP (nitrogen dioxide $\left(\mathrm{NO}_{2}\right)$ and distance to major roads) and wheeze, asthma prevalence and lung function were investigated in participants of the Tasmanian Longitudinal Health Study at 45- and 50-year follow-ups. Generalised estimating equations were used to quantify associations and the potential modifying effect of glutathione $S$-transferase gene variants.

Living $<200 \mathrm{~m}$ from a major road was associated with increased prevalence of current asthma and wheeze, and lower lung function. The association between living $<200 \mathrm{~m}$ from a major road and current asthma and wheeze was more marked for carriers of the GSTT1 null and GSTP1 val/val or ile/val genotypes. Over the 5-year period, higher $\mathrm{NO}_{2}$ exposures were associated with increased current asthma prevalence. Higher $\mathrm{NO}_{2}$ exposure was associated with lower forced vital capacity for carriers of the GSTT1 null genotype.

TRAP exposures were associated with increased risk of asthma, wheeze and lower lung function in middle-aged adults. The interaction with the GSTT1 genotype suggests that deficient antioxidant mechanisms may play a role in these adverse health effects.

This article has supplementary material available from erj.ersjournals.com

Received: Dec 012016 | Accepted after revision: July 232017

Conflict of interest: Disclosures can be found alongside this article at erj.ersjournals.com

Copyright OERS 2017 
Affiliations: ${ }^{1}$ Allergy and Lung Health Unit, Centre for Epidemiology and Biostatistics, School of Population and Global Health, University of Melbourne, Melbourne, Australia. ${ }^{2}$ School of Psychology and Public Health, Dept of Public Health, Latrobe University, Melbourne, Australia. ${ }^{3}$ Murdoch Childrens Research Institute, Melbourne, Australia. ${ }^{4}$ School of Public Health, University of Queensland, Herston, Australia. ${ }^{5}$ Woolcock Institute of Medical Research, University of Sydney, Sydney, Australia. ${ }^{6}$ South Western Sydney Clinical School, UNSW Australia, Sydney, Australia. ${ }^{7}$ POWHCS and IIRC, Faculty of Medicine, University of New South Wales, Sydney, Australia. ${ }^{8}$ NHMRC CRE, University of Tasmania Medical School, Hobart, Australia. ${ }^{9}$ Cancer Epidemiology Centre, Cancer Council Victoria, Melbourne, Australia. ${ }^{10}$ School of Public Health and Preventive Medicine, Monash University, Melbourne, Australia. ${ }^{11}$ Busselton Population Medical Research Institute, Perth, Australia. ${ }^{12}$ School of Population Health, University of Western Australia, Perth, Australia. ${ }^{13}$ School of Pathology and Laboratory Medicine, University of Western Australia, Perth, Australia. ${ }^{14}$ PathWest Laboratory Medicine WA, Sir Charles Gairdner Hospital, Perth, Australia. ${ }^{15}$ Graduate Entry Medical School, University of Limerick, Limerick, Ireland. ${ }^{16}$ Equal senior authors.

Correspondence: Shyamali C. Dharmage, Allergy and Lung Health Unit, Centre for Epidemiology and Biostatistics, School of Population and Global Health, University of Melbourne, L3, 207 Bouverie Street, Carlton, Victoria 3053, Australia. E-mail: s.dharmageđunimelb.edu.au

\section{Introduction}

Asthma in adults is a common and important public health problem. It is a heterogeneous disease that may appear at any age and shows variable activity over time [1]. The rapidly increasing prevalence of asthma after the second half of the twentieth century strongly suggests that environmental exposures play a major role. In particular, the role of traffic-related air pollution (TRAP) exposures in exacerbating or causing asthma has attracted substantial interest [2].

The role of short-term (timescales of days to months) TRAP exposure and respiratory health in adults has been the subject of multiple investigations. However, few studies have investigated the effects of long-term (over years to decades) TRAP exposure on asthma risk [3-5] and reduced lung function [6, 7]. The studies that have been conducted have produced inconsistent results. A multicentre analysis of the European Study of Cohorts for Air Pollution Effects (ESCAPE) found no association between exposure to nitrogen dioxide $\left(\mathrm{NO}_{2}\right)$, particular matter $<2.5 \mu \mathrm{m}(\mathrm{PM} 2.5)$ or particular matter $<10 \mu \mathrm{m}$ (PM10) and longitudinal change in lung function [6]. In contrast, a US study found that living $<100 \mathrm{~m}$ from a major road and increased PM2.5 exposure were both associated with greater lung function decline [7]. A nationwide study of US women reported that long-term PM2.5 exposure was associated with asthma [3]. The Swiss Study on Air Pollution and Lung Disease in Adults (SAPALDIA) found that reduction in PM10 over 12 years was associated with reduced wheeze [8]. Conversely, a study of Australian women similarly found no association between $\mathrm{NO}_{2}$ and asthma [4].

It is possible that differences in genetic susceptibility may, at least in part, explain these apparently inconsistent findings. Components of TRAP, such as $\mathrm{NO}_{2}$ and $\mathrm{PM} 2.5$, cause oxidative stress and subsequent damage to the airways, leading to inflammation and lung remodelling [9]. Oxidative stress generated by exogenous sources is buffered by enzymatic and nonenzymatic antioxidants [10]. Variants of the glutathione S-transferase (GST) $\pi 1, \mu 1$ and $\theta 1$ genes (GSTP1, GSTM1 and GSTT1, respectively) are of considerable interest, given that these genes control enzymes involved in the regulation of oxidative stress by detoxifying reactive oxygen species (ROS) [11]. Our previous cross-sectional study found that mean annual $\mathrm{NO}_{2}$ exposure increased the risk of current asthma, wheeze and allergic sensitisation, and that these effects were modified by GSTT1 polymorphisms, with null carriers at increased risk [12]. SAPALDIA found that decreased PM10 exposure over 11 years was associated with an attenuated decline of forced expiratory flow at $25-75 \%$ of forced vital capacity (FVC) in carriers of GSTP1 val/val. However, they did not find significant interactions with GSTM1, GSTT1 or other lung function outcomes [13].

In this study, our aim was to investigate the effects of TRAP exposure measured using $\mathrm{NO}_{2}$ and living closer to major roads in two follow-ups over a 5-year period on the prevalence of middle-age current asthma, current wheeze and lung function measured in both follow-ups. We also investigated whether these associations were modified by GST gene polymorphisms.

\section{Methods \\ Study population}

The study sample consisted of participants in the 45- and 50-year follow-ups of the Tasmanian Longitudinal Health Study (TAHS) [14-16]. Briefly, the TAHS included children born in 1961 ( $\mathrm{n}=8583)$ and schooled in Tasmania, Australia in 1968. In 2002-2005, the majority of the cohort (67\%) was retraced and resurveyed. Respondents to this survey who had either participated in past follow-up studies and/or reported symptoms of asthma or cough were invited to participate in a laboratory study in 2005-2008 
$(\mathrm{n}=2387)$. Of those invited, $1397(58.6 \%)$ took part in a full laboratory visit and completed a questionnaire, and $354(14.8 \%)$ completed a telephone questionnaire or laboratory visit only. All participants who took part in the full laboratory study $(n=1397)$ were invited for another laboratory study during 2010 and 2012. Of those, 794 (56.8\%) took part in a full laboratory visit and completed a questionnaire, and 43 (3.1\%) completed a telephone questionnaire or laboratory visit only. Although the TAHS started in the state of Tasmania, the study participants are now distributed throughout Australia [16]. The mean \pm SD age of participants was $44.8 \pm 1$ and $49.6 \pm 0.6$ years at the 2005-2008 and 2010-2012 follow-ups, respectively. We investigated the association between TRAP exposure and respiratory health of participants who completed both the 2005-2008 study (referred to as the 45-year follow-up) and the 2010-2012 study (referred to as the 50-year follow-up). Addresses were successfully geocoded for 780 out of the 837 (93.2\%) eligible participants. The 45- and 50-year follow-ups were approved by the Human Research Ethics Committee of the University of Melbourne, Melbourne, Australia (040375.1). Written informed consent was provided by all participants.

\section{TRAP exposure assessment}

Living <200 $m$ from a major road

The distance from each participant's residence to the nearest major road at age 45 and 50 years was calculated using ArcGIS version 10.1 (Environmental Systems Research Institute, Redlands, CA, USA). Major roads were defined using Australian transport hierarchy codes supplied by the Public Sector Mapping Agencies [17]. Codes 301 and 302 were used to identify major roads in the states where participants lived (Victoria, Tasmania, Queensland and New South Wales). Participants were categorised into two groups based on the observation that most components of TRAP decay towards near-background levels within $\sim 200 \mathrm{~m}$ downwind of major roads [18]: 1) living <200 $\mathrm{m}$ and 2) living $>200 \mathrm{~m}$ from a major road.

\section{$\mathrm{NO}_{2}$ exposure}

Mean annual $\mathrm{NO}_{2}$ exposures were assigned using a satellite-based land-use regression (LUR) model [19]. Briefly, this LUR model estimated mean annual $\mathrm{NO}_{2}$ levels based on tropospheric $\mathrm{NO}_{2}$ columns observed by satellite in combination with other predictors, such as land use and roads, to estimate ground-level $\mathrm{NO}_{2}$ across Australia. The previously published satellite-based LUR model used in this study was internally cross-validated using 68 monitoring sites across Australia, including all states and territories (apart from the sparsely populated Northern Territory). The model captured $81 \%$ of spatial variability in annual $\mathrm{NO}_{2}$, with a cross-validated prediction error of 19\% [19]. We also conducted an external validation for our LUR model; it captured $66 \%$ of annual $\mathrm{NO}_{2}$ at a completely independent set of 98 urban background and near-traffic validation sites across Australia. Mean prediction bias was low $(-0.2 \mathrm{ppb})$ and prediction error was comparable to our initial cross-validation results (19\% versus $25 \%$ in the original and validated results, respectively) [12]. The spatial resolution of our LUR model was variable, as the LUR predictors also varied in resolution. In practice, this resulted in an approximate resolution of 150-200 $\mathrm{m}$ in urban areas and $1 \mathrm{~km}$ in nonurban areas. The spatial variability of $\mathrm{NO}_{2}$ is greater in urban areas and $\sim 80 \%$ of the TAHS participants in this study lived in "significant urban areas", as defined by the Australian Bureau of Statistics [20]. Mean annual residential exposures to outdoor $\mathrm{NO}_{2}$ at age 45 and 50 years were estimated and assigned separately based on participants' geocoded addresses. Therefore, each participant had separate annual average $\mathrm{NO}_{2}$ values at age 45 and 50 years. Consequently, the exposure (mean $\mathrm{NO}_{2}$ in the year leading up to respiratory measurement) was measured and recorded before the outcome at both time-points.

\section{Pre-bronchodilator spirometry}

Pre-bronchodilator spirometry at both time-points was performed with the EasyOne Ultrasonic Spirometer (ndd Medizintechnik, Zurich, Switzerland) using the same methods. Spirometry was conducted according to the guidelines of the American Thoracic Society and European Respiratory Society [21]. Subsequently, Global Lung Initiative 2012 reference values were used to derive z-scores [22]. The z-scores represent the deviation from the age-, sex-, height- and race-adjusted population mean in standard deviation units.

\section{Definitions of current asthma and wheeze}

Current asthma at 45 and 50 years was defined as having asthma or wheezy breathing within the last 12 months. Current wheeze at both follow-ups was defined as wheezing or whistling in the chest without having had a cold in the last 12 months.

\section{Genetic data}

Blood samples for genetic analysis were collected at the 45-year follow-up. Genotypes of GSTM1 null and GSTT1 null were detected using a multiplex PCR technique [23], and for all experiments positive primers 
for $\beta$-globin were included as a positive PCR control. Individuals were categorised for GSTT1 as either GSTT1 null (homozygous for the GSTT1*0 allele) or GSTT1 non-null (homozygous or heterozygous for the $G S T T 1^{\star} 1$ allele). For GSTM1, individuals were categorised as either GSTM1 null (homozygous for the $G S T M 1{ }^{\star} 0$ allele) or GSTM1 non-null (possessing at least one functional GSTM1 allele). A customised GoldenGate genotyping assay (Illumina, San Diego, CA, USA) was used to genotype the GSTP1 (rs1695 $\mathrm{A} \rightarrow \mathrm{G}$ : Ile105Val) polymorphism. Individuals were categorised by genotypes as GSTP1-AA (ile/ile), GSTP1-AG (ile/val) or GSTP1-GG (val/val).

\section{Statistical analysis}

TRAP exposure and outcomes at age 45 and 50 years

We investigated associations between $\mathrm{NO}_{2}$ exposure and living $<200 \mathrm{~m}$ from a major road at both age 45 and 50 years (selected based on availability of both exposure and outcome data for this cohort) and outcomes measured at the same ages using a multivariable logistic regression model. Since most participants contributed data at two occasions, we fitted the model using generalised estimating equations (GEEs) with an exchangeable correlation structure to account for nonindependence of outcome data due to repeated measures. More formally, the GEE approach assumes a linear relationship between the log odds of the outcome and exposures. However, it uses a "working" correlation matrix to re-weight the contribution of observations during the model-fitting and estimation process to reflect, in this case, the paired nature of the data and to ensure that correct standard errors are obtained. Assuming independence of observations will understate the true standard errors since, due to within-person correlation of outcomes, two sets of measurements on each of $n$ participants has less information than a single set of measurements on each of $2 \times n$ participants.

We analysed both z-scores of lung function and raw lung function adjusted for age, sex and height.

In this analysis, we only focused on participants who lived at the same address at both time-points (referred to as "nonmovers"), which included $>90 \%(n=709)$ of participants. A sensitivity analysis of those who moved (referred to as "movers") showed negligible variation in estimated effects (data not shown).

\section{GST interactions}

To investigate potential effect modification by genetic polymorphisms, categorical variables representing GST genotypes were added as interaction terms to the GEE models. For all three genes, an autosomal dominant genetic model was assumed, with genotypes entered as binary variables. Interactions with $\mathrm{p} \leqslant 0.10$ were considered significant and stratified analyses were subsequently performed.

In all models, $\mathrm{NO}_{2}$ exposure was entered as a continuous variable and living $<200 \mathrm{~m}$ from a major road as a binary variable. Results for categorical outcomes were reported as odds ratios with $95 \%$ confidence intervals. The results for $\mathrm{NO}_{2}$ exposure were scaled to an interquartile range (IQR) increase in mean annual $\mathrm{NO}_{2}$ at the 50 -year follow-up, which in this sample was $2.4 \mathrm{ppb}$. A directed acyclic graph produced using DAGitty software [24] was constructed to identify possible confounders. This showed what adjustment was required in the regression models in order for the estimated measures of association to have a causal interpretation (under the assumption that the resulting model was correctly specified). Socioeconomic status (defined using educational attainment), smoking status, type of cooking (gas and electric), type of heating (wood/coal, gas room heating and other (electric, ducted heating and reverse cycle air conditioning)) and rural or urban location (using the accessibility/remoteness index of Australia 2006 [21]) were included in the models. The same confounders that were included in the main models were adjusted for in the interaction models. In a sensitivity analysis we included sampling weights and all the other cofounding variables described previously in the model to test whether the findings were influenced by the sampling. Sampling weights were derived by calculating the inverse probability of selection for the 45-year follow-up. All statistical analyses were performed using Stata version 13.1 (StataCorp, College Station, TX, USA).

\section{Results}

Study characteristics were, for the most part, similar between participants in the 45- and 50-year follow-ups, with the exception that those in the 50-year follow-up were less likely to be current smokers and less likely to have asthma (table 1). In this cohort, participants used a number of different methods for cooking and heating. At age 50 years, the use of wood/coal for heating was reduced by nearly $10 \%$ compared with age 45 years and the use of "clean methods for heating" (i.e. electric, ducted heating and reverse cycle air conditioning) increased by $12.4 \%$. From age 45 to 50 years, the use of gas for cooking increased by $6.6 \%$ (table 1 ).

Of the 837 who participated in both the 45- and 50-year follow-ups, 768 (91.2\%) had geocoded address data at the two time-points (figure 1). Of those, a similar proportion lived $<200 \mathrm{~m}$ from a major road at 
both follow-ups (table 2). The mean difference between $\mathrm{NO}_{2}$ exposure in participants living $<200$ versus $>200 \mathrm{~m}$ from a major road was statistically significant, while the participants living $<200 \mathrm{~m}$ from a major road had a higher exposure. Similar significant differences were observed at both age 45 and 50 years ( $t$-test, $\mathrm{p}<0.0001$ ) (supplementary table S2).

Effect of $\mathrm{NO}_{2}$ exposure and living $<200 \mathrm{~m}$ from a major road from age 45 to 50 years on current asthma, current wheeze and lung function

In the GEE models, living $<200 \mathrm{~m}$ from a major road and exposure to an IQR increase in average annual $\mathrm{NO}_{2}$ exposure (i.e. $2.4 \mathrm{ppb}$ ) was associated with increased current asthma prevalence (adjusted OR (aOR) 1.49, 95\% CI 1.09-2.05 and aOR 1.10, 95\% CI 0.96-1.27, respectively). Similar results were observed for living $<200 \mathrm{~m}$ from a major road and increased current wheeze prevalence (aOR 1.61, 95\% CI 1.19-2.19). Living $<200 \mathrm{~m}$ from a major road was also associated with lower forced expiratory volume in $1 \mathrm{~s}(\mathrm{FEV} 1)(\beta$ $-0.32,95 \%$ CI $-0.49--0.15)$, FVC $(\beta-0.20,95 \%$ CI $-0.35--0.05)$ and FEV $1 / F V C(\beta-0.19,95 \%$ CI $-0.34-0.04$ ) (table 3). We observed similar associations with raw lung function adjusted for age, sex and height (supplementary table S1). These findings remained similar in the sensitivity analysis that adjusted for sampling weights (supplementary table S2).

Influence of GST gene polymorphisms on the relationship between TRAP exposure from age 45 to 50 years and current asthma, current wheeze and lung function

Polymorphisms of GSTT1 were found to modify the associations between TRAP exposure from age 45 to 50 years and respiratory outcomes. $\mathrm{NO}_{2}$ exposure from age 45 to 50 years was associated with a significant reduction in FEV1 and a trend towards reduction in FVC only for GSTT1 null carriers ( $\mathrm{p}_{\text {interaction }}=0.01$ and 0.05 , respectively).

TABLE 1 Study characteristics of the 709 participants who had a geocoded residential address at age 45 and 50 years

\begin{tabular}{|c|c|c|}
\hline & Age 45 years & Age 50 years \\
\hline Male & $345(48.7)$ & $345(48.7)$ \\
\hline \multicolumn{3}{|c|}{ Socioeconomic status (education) } \\
\hline Grade 1-9 & $38(5.4)$ & $38(5.4)$ \\
\hline Grade 10 or 12 & 264 (37.3) & 264 (37.3) \\
\hline Trade/apprenticeship & $252(35.6)$ & $252(35.6)$ \\
\hline University degree or higher & $153(21.6)$ & $153(21.6)$ \\
\hline \multicolumn{3}{|l|}{ Smoking status } \\
\hline Never-smoker & $318(45)$ & 309 (43.9) \\
\hline Ex-smoker & 226 (31.9) & $266(37.8)$ \\
\hline Current smoker & $163(23.1)$ & 129 (18.3) \\
\hline \multicolumn{3}{|l|}{ Type of cooking } \\
\hline Gas & $141(20.1)$ & $185(26.7)$ \\
\hline Electric & $562(79.9)$ & 507 (73.3) \\
\hline \multicolumn{3}{|l|}{ Type of heating } \\
\hline Wood/coal & $320(45.4)$ & 243 (34.5) \\
\hline Gas room heater & $60(8.5)$ & $49(7.1)$ \\
\hline Other & $325(46.1)$ & $412(58.4)$ \\
\hline Lived in rural areas & $80(11.3)$ & $80(11.3)$ \\
\hline \multicolumn{3}{|l|}{ Respiratory outcomes } \\
\hline Current asthma & $187(27.1)$ & $164(23.4)$ \\
\hline Current wheeze & $209(30.0)$ & $202(28.9)$ \\
\hline \multicolumn{3}{|l|}{ Gene frequencies } \\
\hline GSTT1 null & $94(15.5)$ & $94(15.5)$ \\
\hline GSTM1 null & $338(55.7)$ & $338(55.7)$ \\
\hline GSTP1 val/val+val/ile & $370(60.1)$ & $370(60.1)$ \\
\hline \multicolumn{3}{|l|}{ Lung function } \\
\hline $\mathrm{FEV}_{1}$ & $-0.36 \pm 1.08$ & $-0.37 \pm 1.12$ \\
\hline FVC & $0.02 \pm 1.01$ & $-0.062 \pm 1.00$ \\
\hline $\mathrm{FEV}_{1} / \mathrm{FVC}$ & $-0.63 \pm 1.03$ & $-0.56 \pm 0.93$ \\
\hline
\end{tabular}

Data are presented as $\mathrm{n}(\%)$ or mean \pm SD. FEV1: forced expiratory volume in $1 \mathrm{~s}$; FVC: forced vital capacity. 


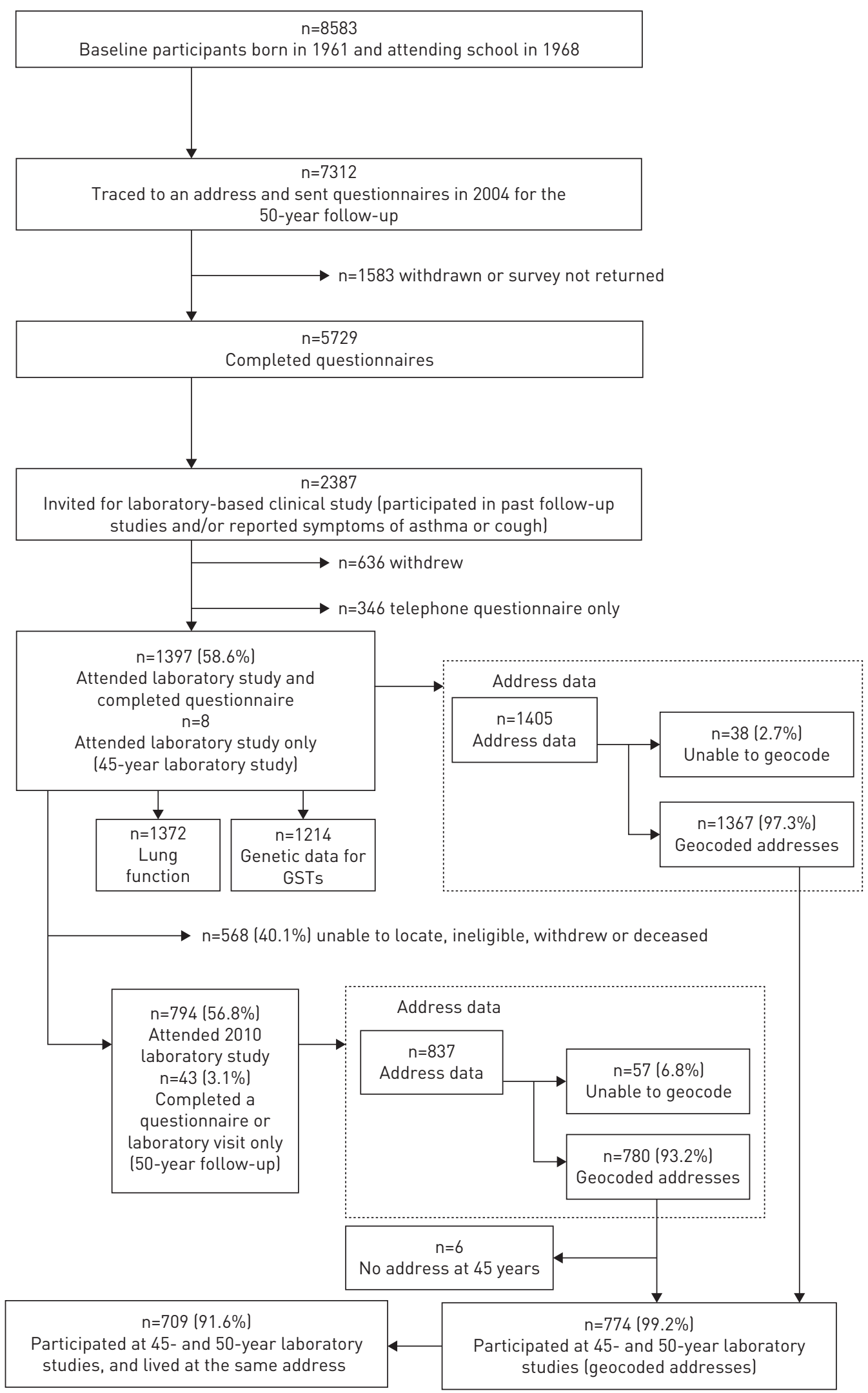

FIGURE 1 Overview of the Tasmania Longitudinal Health Study follow-ups [16]. 
TABLE 2 Traffic-related air pollution exposure variables for the 709 participants who had a geocoded residential address at age 45 and 50 years

\begin{tabular}{lcc} 
& Age $\mathbf{4 5}$ years & Age $\mathbf{5 0}$ years \\
\hline Residential address $<\mathbf{2 0 0}$ m from a major road & $198(28.0)$ & $196(27.64)$ \\
Mean annual nitrogen dioxide exposure $\mathbf{p p b}$ & & \\
Quartile 1 & 3.7 & 3.1 \\
Quartile 2 & 4.6 & 3.9 \\
Quartile 1 & 6.1 & 5.4 \\
Mean \pm SD & $5.4 \pm 2.8$ & $4.6 \pm 2.6$ \\
Lowest & 2.5 & 1.8 \\
Highest & 23.8 & 22.9 \\
\hline
\end{tabular}

Data are presented as $\mathrm{n}(\%)$, unless otherwise stated.

GSTT1 polymorphisms were found to significantly modify the association between living $<200 \mathrm{~m}$ from a major road and risk of current asthma and wheeze. The associations between living $<200 \mathrm{~m}$ from a major road and increased risk of current asthma and wheeze were more marked in carriers of the GSTT1 null genotype (aOR 2.84, 95\% CI 1.15-7.01 and aOR 3.19, 95\% CI 1.15-8.77, respectively).

GSTP1 showed a significant interaction with $\mathrm{NO}_{2}$ exposure from age 45 to 50 years and current asthma and wheeze prevalence $\left(p_{\text {interaction }}=0.06\right.$ and 0.02 , respectively), with carriers of GSTP1 val/val or ile/val having an increased current asthma prevalence when exposed to elevated levels of $\mathrm{NO}_{2}$. There was an unexpected significant interaction observed for GSTM1 in the association between living $<200 \mathrm{~m}$ from a

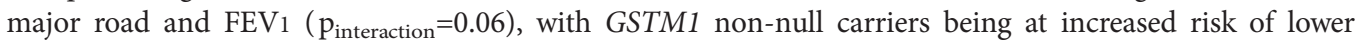
FEV1 (table 4).

\section{Discussion}

Our study provides evidence that long-term TRAP exposure, as measured by $\mathrm{NO}_{2}$ and living $<200 \mathrm{~m}$ from a major road, is associated with current asthma. Additionally, living $<200 \mathrm{~m}$ from a major road over this same period is associated with a lower FEV1, FVC and FEV1/FVC in adults aged 45-50 years. GSTT1 and GSTP1 polymorphisms modify the effects of living $<200 \mathrm{~m}$ on the prevalence of current asthma and wheeze, and also modify the effect of changes in $\mathrm{NO}_{2}$ exposure on FVC, with GSTT1 null genotype carriers at an increased risk.

TABLE 3 Effect of traffic-related air pollution exposure (nitrogen dioxide $\left(\mathrm{NO}_{2}\right)$ exposure and living $<200 \mathrm{~m}$ from a major road from age 45 to 50 years) on current asthma, current wheeze and lung function (restricted to the 709 nonmovers)

\begin{tabular}{|c|c|c|c|c|c|c|c|c|}
\hline & \multicolumn{2}{|c|}{ Unadjusted } & \multicolumn{2}{|c|}{ Adjusted $^{\#}$} & \multicolumn{2}{|l|}{ Unadjusted } & \multicolumn{2}{|l|}{ Adjusted $^{\#}$} \\
\hline \multicolumn{9}{|l|}{$\mathrm{NO}_{2}$ exposure } \\
\hline Current asthma & $1.07(0.95-1.21)$ & 0.26 & $1.10(0.96-1.27)$ & 0.15 & & & & \\
\hline Current wheeze & $0.98(0.87-1.11)$ & 0.78 & $1.05(0.91-1.20)$ & 0.50 & & & & \\
\hline $\mathrm{zFEV}_{1}{ }^{\Uparrow}$ & & & & & $0.00(-0.06-0.06)$ & 0.98 & $-0.02(-0.09-0.04)$ & 0.47 \\
\hline zFVC & & & & & $0.03(-0.03-0.09)$ & 0.28 & $0.00(-0.06-0.07)$ & 0.91 \\
\hline Current asthma & $1.48(1.08-2.02)$ & 0.01 & $1.49(1.09-2.05)$ & 0.01 & & & & \\
\hline Current wheeze & $1.53(1.13-2.05)$ & 0.01 & $1.61(1.19-2.19)$ & $<0.01$ & & & & \\
\hline $\mathrm{zFEV}_{1}{ }^{\Uparrow}$ & & & & & $-0.27(-0.44--0.10)$ & $<0.01$ & $-0.32(-0.49--0.15)$ & $<0.01$ \\
\hline zFVcף & & & & & $-0.16(-0.32--0.01)$ & 0.04 & $-0.20(-0.35--0.05)$ & 0.01 \\
\hline $\mathrm{zFEV}_{1} / \mathrm{FVC}^{\text {" }}$ & & & & & $-0.16(-0.31--0.01)$ & 0.03 & $-0.19(-0.34--0.04)$ & 0.01 \\
\hline
\end{tabular}


TABLE 4 Associations between traffic-related air pollution exposure (nitrogen dioxide $\left(\mathrm{NO}_{2}\right)$ exposure and $<200 \mathrm{~m}$ from a major road from age 45 to 50 years) and current asthma and current wheeze and lung function, stratified by glutathione S-transferase genotype (restricted to the 709 nonmovers)

\begin{tabular}{|c|c|c|c|c|}
\hline Respiratory outcome & Gene variation & $\mathbf{p}_{\text {interaction }}-$ value & $\mathrm{OR} / \beta^{\#}(95 \% \mathrm{CI})$ & p-value \\
\hline \multicolumn{5}{|l|}{$\mathrm{NO}_{2}$ exposure } \\
\hline $\mathrm{zFEV}_{1}^{+}$ & GSTT1 null & 0.05 & $-0.12(-0.41-0.02)$ & 0.06 \\
\hline \multirow[t]{2}{*}{$\mathrm{zFVC}^{+}$} & GSTT1 null & 0.01 & $-0.21(-0.42--0.01)$ & 0.04 \\
\hline & GSTT1 non-null & & $0.04(-0.03-0.12)$ & 0.22 \\
\hline Asthma & GSTP1 ile/ile & 0.06 & $0.91(0.71-1.15)$ & 0.42 \\
\hline Wheeze & GSTP1 val/val+ile/val & & $1.18(0.97-1.42)$ & 0.10 \\
\hline \multicolumn{5}{|c|}{ Living $<200 \mathrm{~m}$ from a major road } \\
\hline \multirow[t]{2}{*}{ Asthma } & GSTT1 null & 0.09 & $2.84(1.15-7.01)$ & 0.02 \\
\hline & GSTT1 non-null & & $1.40(0.95-2.05)$ & 0.09 \\
\hline \multirow[t]{2}{*}{ Wheeze } & GSTT1 null & 0.10 & $3.19(1.15-8.77)$ & 0.02 \\
\hline & GSTT1 non-null & & $1.44(1.00-2.08)$ & 0.05 \\
\hline
\end{tabular}

Australia is a continent with relatively low levels of air pollution [25] and the current national air quality standard for annual mean $\mathrm{NO}_{2}$ is $30 \mathrm{ppb}$ [26]. In our current study, the maximum annual mean $\mathrm{NO}_{2}$ exposure for participants over the two time-points was estimated at 23.8 and $22.9 \mathrm{ppb}$ at age 45 and 50 years, respectively. The mean $\pm \mathrm{SD} \mathrm{NO}_{2}$ exposure at age 45 and 50 years for the study population was 5.4 \pm 2.6 and $4.6 \pm 2.6 \mathrm{ppb}$. Our results indicate that long-term exposure to low levels of $\mathrm{NO}_{2}$, even levels well below the Australian standards, was associated with increased risk of respiratory diseases. In our previous cross-sectional study, we showed that annual $\mathrm{NO}_{2}$ exposure or living $<200 \mathrm{~m}$ from a major road was associated with current asthma, wheeze, allergic sensitisation and lower levels of lung function, and we also found significant interactions with GSTT1 [27]. This adds to the body of evidence showing that both short- and long-term TRAP exposures, even at low levels of pollution, contribute to adverse effects on health. This is especially the case for genetically susceptible populations.

We observed associations with low $\mathrm{NO}_{2}$ exposure and asthma. There is evidence from other countries that low levels of $\mathrm{NO}_{2}$ are associated with respiratory diseases. For example, the SAPALDIA study found a mean annual average of $18.92 \mathrm{ppb}\left(35.6 \mu \mathrm{g} \cdot \mathrm{m}^{-3}\right)$ associated with wheeze in smokers [5]. The ESCAPE study, combining six European cohorts, found positive associations between $\mathrm{NO}_{2}$ exposure and incidence of asthma in adults, where mean annual $\mathrm{NO}_{2}$ concentrations ranged from 11.6 to $16.4 \mathrm{ppb}$ [28]. It has been shown that air pollution exposure and adverse health outcomes follow a linear dose-response relationship. Therefore, adverse health outcomes associated with lower air pollution levels are to be expected.

Our finding of an association between residing closer to a major road and reduced lung function confirms findings from the Framingham study and a German study on the influence of air pollution on lung function, inflammation and ageing in women (SALIA) [7, 29]. We did not see similar effects with long-term $\mathrm{NO}_{2}$ exposure, whereas the ESCAPE, SALIA and SAPALDIA studies all reported that this exposure was associated with reduced lung function $[6,29,30]$. The null findings in our study may be due to consistently low levels of $\mathrm{NO}_{2}$ exposure over the 5-year period (mean $\pm \mathrm{sD} \mathrm{NO} \mathrm{NO}_{2} 5.0 \pm 2.6 \mathrm{ppb}$ ). The three aforementioned studies reported a mean $\mathrm{NO}_{2}$ exposure between 12 and $20 \mathrm{ppb}$, which was more than double that observed in our study. Additionally, their larger sample sizes, compared with ours, may have provided more power to detect associations.

There is evidence from human and animal toxicology studies that release of ROS triggered by air pollution exposure is an important step in the pathway leading to oxidative damage and inflammation [31]. However, the biological mechanisms behind long-term air pollution exposure and deteriorating adult lung 
function have not been well characterised in humans. Experimental studies provide evidence that short-term exposure to $\mathrm{NO}_{2}$ and particulate matter can induce endogenous release of ROS in exposed lung epithelial cells. This leads to allergic responses, pulmonary inflammation and pulmonary remodelling [32-34].

Variants of the GSTP1, GSTM1 and GSTT1 genes act through a common mechanism, protecting exposed tissues from the oxidative damage that may be caused by ROS, by conjugating them with glutathione [11]. Our study is the first to investigate whether GSTs modify the association between long-term air pollution exposure and respiratory or lung function outcomes. Previously, using a cross-sectional study, we showed that living $<200 \mathrm{~m}$ from a major road for a short period of time was associated with increased risk of current asthma and wheeze for carriers of GSTT1 null [12]. In the current analysis, we were able to show similar results with asthma and wheeze for those who lived $<200 \mathrm{~m}$ from a major road.

A recent controlled chamber study of human subjects demonstrated that short-term exposure to diesel exhaust particles (DEPs) augmented allergen-induced markers of both allergic and nonallergic inflammation [34]. The authors found that having GSTT1 null enhanced those end-points [34]. In our analysis, we did not have data on DEPs, but previous literature has shown that both $\mathrm{NO}_{2}$ and distance to major roads are proxies for the complex mixture represented by TRAP, which includes DEPs [18]. Thus, similar types of mechanisms are expected to be involved in TRAP exposure and airway inflammation for GSTT1 null carriers. For the GSTP1 gene, the identification of the risk allele is not clear. Some studies have shown that risk increases for those with the ile allele, while others have shown that val allele increases the risk $[13,35]$. Similarly, previous studies have shown that GSTM1 non-null carriers have an increased risk of respiratory diseases $[27,36]$. Apart from GSTs, polymorphisms in other oxidative stress genes have been shown to interact with the association of TRAP exposure and asthma. For example, a study by CASTRO-GINER et al. [37] showed that polymorphisms of NQO1 (NADPH quinone oxidoreductase 1) or TNFA (tumour necrosis factor- $\alpha$ ) were associated with increased susceptibility to $\mathrm{NO}_{2}$ exposure and asthma in adults.

Our study has both strengths and limitations. The TAHS is a well-characterised longitudinal study including repeated measures of lung function and prospectively collected data on a range of risk factors. The average follow-up period for the current analysis was 5 years. Similar studies investigating long-term air pollution exposure have used longer follow-ups [3, 7]. Additionally, in our cohort, address data was collected at age 45 and 50 years, but not the years in between. This may lead to exposure misclassification of long-term exposures, but few participants had moved and $\mathrm{NO}_{2}$ exposure was very similar between movers and nonmovers. We used modelled $\mathrm{NO}_{2}$ exposure from a LUR model for all participants regardless of the location. The LUR model has been found to predict annual $\mathrm{NO}_{2}$ exposure with a relatively low error of $19 \%$ [19]. However, we cannot ascribe our findings to $\mathrm{NO}_{2}$ specifically as it is only one proxy for TRAP. Previous studies investigating long-term TRAP exposure have used various methods to define "long-term exposure". LAZAREvic et al. [4] used 3-year average $\mathrm{NO}_{2}$ to investigate asthma symptom prevalence. A study combining six European cohorts estimated $\mathrm{NO}_{2}$ exposures using a LUR model at 2010 or 2011, although some of the cohorts included in this study were initiated well before the air pollution modelling was conducted [28]. Similarly, three studies (a nationwide cohort of US women, a longitudinal study of the European Respiratory Health Survey and Respiratory Health in Northern Europe (RHINE)) used annual average $\mathrm{NO}_{2}$ exposure in a single year $[3,38,39]$. In all these studies, exposure measurements were inconsistent when examining long-term exposures. In our analysis we assigned $\mathrm{NO}_{2}$ exposure separately at two follow-ups, representing actual time-points where the outcome data were collected.

Using distance to major roads as a proxy for TRAP exposure also has limitations. Living closer to a major road alone does not account for complex factors such as wind direction, traffic volume and composition (e.g. heavy diesel versus light passenger vehicles) that can contribute to TRAP exposure in a certain geographic location. Although there are a number of limitations in using distance to major roads as a proxy for TRAP exposure, our study found consistent and significant associations with living <200 $\mathrm{m}$ from a major road. Previous work demonstrates that freshly emitted pollutants and resuspended particles show strong spatial gradients near major roads, with an approximately exponential decay in concentrations to background levels occurring within $\sim 300-500 \mathrm{~m}$ [40]. Our selection of a $200 \mathrm{~m}$ distance threshold from a major road was consistent with previous studies [41, 42] and informed by the Special Report of the Health Effects Institute [43]. In this study, we observed stronger associations between our outcomes and living near a major road, compared with LUR-based estimates of $\mathrm{NO}_{2}$. While $\mathrm{NO}_{2}$ is a useful proxy of combustion sources, distance to a major road may better capture other important pollutants such as black carbon, fine (PM2.5) and ultrafine particles, and volatile organic compounds as a whole mixture, even if it is a relatively crude measure of TRAP. Moreover, LUR models are not capable of distinguishing aged $\mathrm{NO}_{2}$ compared with freshly produced $\mathrm{NO}_{2}$ from traffic emissions. 
Although we investigated long-term associations using GEE models, there is uncertainty in the degree to which exposure precedes outcomes observed in this group of adults. The longer-term associations observed might result from some cumulative exposure over time. There was a high degree of colinearity in TRAP exposures at age 45 and 50 years, and therefore it was difficult to untangle whether the observed outcomes were purely based on long-term or cross-sectional associations. It should also be noted that the current study included a subsample of the 2002-2005 follow-up, a survey of all the cohort members who were traced to an address. The selected subsample from the 2002-2005 follow-up, when participants were 45 years old, was enriched for symptoms of asthma or cough. This subsample was then invited to participate in another laboratory study when they were 50 years old. As a result, this sample has a higher prevalence of asthma. However, in our sensitivity analysis there was no difference in obtained results after controlling for sampling weights. This indicates that the observed associations did not change based on the selection of participants in this study.

In conclusion, in our study, even relatively low levels of $\mathrm{NO}_{2}$ exposure in adults were found to be associated with increased current asthma prevalence. Similarly, living $<200 \mathrm{~m}$ from a major road from age 45 to 50 years was found to be associated with increased risk of current asthma, current wheeze and lower lung function. Carriers of the GSTT1 null genotype may be at a greater risk for asthma and wheeze if they live for a longer period of time closer to a major road $(<200 \mathrm{~m})$. This provides evidence at the population level that genetic polymorphisms associated with the antioxidant defence system are important in regulating the harmful effects of TRAP exposure. Our study adds to the existing body of evidence that even relatively low levels of TRAP exposure are associated with asthma and poor lung function in adults.

\section{Acknowledgements}

The authors thank all the Tasmania Longitudinal Health Study participants, staff and funding bodies. We are grateful to Adrian Lowe (University of Melbourne and Murdoch Childrens Research Institute, Melbourne, Australia) for his valuable discussions and feedback. G. Bowatte, L.D. Knibbs and J.L. Perret are supported by the Centre for Air Quality and Health Research and Evaluation (CAR), a National Health and Medical Research Council-funded centre for research excellence.

Authors contributions: Acquisition of data: S.C. Dharmage, E.H. Walters, M.C. Matheson, M.J. Abramson, C.J. Lodge, P.S. Thomas, D.P. Johns, L.C. Gurrin, G.G. Giles, J.L. Perret, J. Hui and L.D. Knibbs. Conception and design: S.C. Dharmage, B. Erbas and G. Bowatte. Drafting the article: G. Bowatte, S.C. Dharmage, C.J. Lodge and B. Erbas. Revision for important intellectual content: S.C. Dharmage, E.H. Walters, M.J. Abramson, M.C. Matheson, L.C. Gurrin, C.J. Lodge, L.D. Knibbs, L.C. Gurrin, G.B. Marks, P.S. Thomas, D.P. Johns, G.G. Giles, J. Hui, J.L. Perret and M. Dennekamp.

\section{References}

Wenzel S. Asthma phenotypes: the evolution from clinical to molecular approaches. Nat Med 2012; 18: 716-725. Eder W, Ege MJ, von Mutius E. The asthma epidemic. N Engl J Med 2006; 355: 2226-2235.

Young MT, Sandler DP, DeRoo LA, et al. Ambient air pollution exposure and incident adult asthma in a nationwide cohort of U.S. women. Am J Respir Crit Care Med 2014; 190: 914-921.

4 Lazarevic N, Dobson AJ, Barnett AG, et al. Long-term ambient air pollution exposure and self-reported morbidity in the Australian Longitudinal Study on Women's Health: a cross-sectional study. BMJ Open 2015; 5: e008714.

5 Zemp E, Elsasser S, Schindler C, et al. Long-term ambient air pollution and respiratory symptoms in adults (SAPALDIA study). The SAPALDIA Team. Am J Respir Crit Care Med 1999; 159: 1257-1266.

6 Adam M, Schikowski T, Carsin AE, et al. Adult lung function and long-term air pollution exposure. ESCAPE: a multicentre cohort study and meta-analysis. Eur Respir J 2015; 45: 38-50.

7 Rice MB, Ljungman PL, Wilker EH, et al. Long-term exposure to traffic emissions and fine particulate matter and lung function decline in the Framingham Heart Study. Am J Respir Crit Care Med 2015; 191: 656-664.

8 Schindler C, Keidel D, Gerbase MW, et al. Improvements in $\mathrm{PM}_{10}$ exposure and reduced rates of respiratory symptoms in a cohort of Swiss adults (SAPALDIA). Am J Respir Crit Care Med 2009; 179: 579-587.

9 Guarnieri M, Balmes JR. Outdoor air pollution and asthma. Lancet 2014; 383: 1581-1592.

10 Birben E, Sahiner UM, Sackesen C, et al. Oxidative stress and antioxidant defense. World Allergy Organ J 2012; 5: 9-19.

11 McCunney R. Asthma, genes, and air pollution. J Occup Environ Med 2005; 47: 1285-1291.

12 Bowatte G, Lodge CJ, Knibbs LD, et al. Traffic-related air pollution exposure is associated with allergic sensitization, asthma, and poor lung function in middle age. J Allergy Clin Immunol 2016; 139: 122-129.

13 Curjuric I, Imboden M, Schindler C, et al. HMOX1 and GST variants modify attenuation of FEF $25-75 \%$ decline due to PM10 reduction. Eur Respir J 2009; 35: 505-514.

14 Wharton C, Dharmage S, Jenkins M, et al. Tracing 8,600 participants 36 years after recruitment at age seven for the Tasmanian Asthma Study. Aust NZ J Public Health 2006; 30: 105-110.

15 Gibson HB, Silverstone H, Gandevia B, et al. Respiratory disorders in seven-year-old children in Tasmania. Aims, methods and administration of the survey. Med J Aust 1969; 2: 201-205.

16 Matheson MC, Abramson MJ, Allen K, et al. Cohort profile: the Tasmanian Longitudinal Health study (TAHS). Int J Epidemiol 2017; 46: 407-408.

17 The Intergovernmental Committee of Surveying and Mapping. Assessing the Feasibility of a National Road Classification. Report to ICSM on National Road Classification Developments. 2006. www.icsm.gov.au/roads/ Assessing_Feasibility_National_Road_Classification.pdf Date last accessed: March 15, 2016. 
18 Karner AA, Elsinger DS, Niemeier DA. Near-roadway air quality: synthesizing the findings from real-world data. Environ Sci Technol 2010; 44: 5334-5344.

19 Knibbs LD, Hewson MG, Bechle MJ, et al. A national satellite-based land-use regression model for air pollution exposure assessment in Australia. Environ Res 2014; 135: 204-211.

20 Australian Bureau of Statistics. Australian Statistical Geography Standard (ASGS): Volume 4: Significant Urban Areas, Urban Centres and Localities, Section of State. Canberra, Australian Bureau of Statistics, 2011.

21 Miller MR, Hankinson J, Brusasco V, et al. Standardisation of spirometry. Eur Respir J 2005; 26: 319-338.

22 Quanjer PH, Stanojevic S, Cole TJ, et al. Multi-ethnic reference values for spirometry for the 3-95-yr age range: the global lung function 2012 equations. Eur Respir J 2012; 40: 1324-1343.

$23 \mathrm{Wu} \mathrm{W}$, Lu J, Tang Q, et al. GSTM1 and GSTT1 null polymorphisms and male infertility risk: an updated meta-analysis encompassing 6934 subjects. Sci Rep 2013; 3: 2258.

24 Textor J, Hardt J, Knüppel S. DAGitty: a graphical tool for analyzing causal diagrams. Epidemiology 2011; 22: 745-745.

25 Janssen NA, Brunekreef B, van Vliet P, et al. The relationship between air pollution from heavy traffic and allergic sensitization, bronchial hyperresponsiveness, and respiratory symptoms in Dutch schoolchildren. Environ Health Perspect 2003; 111: 1512-1518.

26 Dept of the Environment and Heritage. National Standards for Criteria Air Pollutants in Australia. 2005. www. environment.gov.au/protection/publications/factsheet-national-standards-criteria-air-pollutants-australia Date last accessed: May 1, 2016.

27 Bowatte G, Lodge CJ, Lowe AJ, et al. Do variants in GSTs modify the association between traffic air pollution and asthma in adolescence? Int J Mol Sci 2016; 17: 485.

28 Jacquemin B, Siroux V, Sanchez M, et al. Ambient air pollution and adult asthma incidence in six European cohorts (ESCAPE). Environ Health Perspect 2015; 123: 613-621.

29 Schikowski T, Sugiri D, Ranft U, et al. Long-term air pollution exposure and living close to busy roads are associated with COPD in women. Respir Res 2005; 6: 152.

30 Ackermann Liebrich U, Leuenberger P, Schwartz J, et al. Lung function and long term exposure to air pollutants in Switzerland. Study on Air Pollution and Lung Diseases in Adults (SAPALDIA) Team. Am J Respir Crit Care Med 1997; 155: 122-129.

31 Huang SK, Zhang Q, Qiu Z, et al. Mechanistic impact of outdoor air pollution on asthma and allergic diseases. J Thorac Dis 2015; 7: 23-33.

32 Hatzis C, Godleski JJ, González-Flecha B, et al. Ambient particulate matter exhibits direct inhibitory effects on oxidative stress enzymes. Environ Sci Technol 2006; 40: 2805-2811.

33 Wang JM. Biochemical and morphological alterations in the lungs and livers of mice following exposure to polluted air in a traffic tunnel. Proc Natl Sci Counc Repub China Part B Life Sci 1992; 16: 77-83.

34 Carlsten C, Blomberg A, Pui M, et al. Diesel exhaust augments allergen-induced lower airway inflammation in allergic individuals: a controlled human exposure study. Thorax 2015; 71: 35-44.

35 Su MW, Tsai CH, Tung KY, et al. GSTP1 is a hub gene for gene-air pollution interactions on childhood asthma. Allergy 2013; 68: 1614-1617.

36 Reddy P, Naidoo RN, Robins TG, et al. GSTM1 and GSTP1 gene variants and the effect of air pollutants on lung function measures in South African children. Am J Ind Med 2012; 55: 1078-1086.

37 Castro-Giner F, Kunzli N, Jacquemin B, et al. Traffic-related air pollution, oxidative stress genes, and asthma (ECHRS). Environ Health Perspect 2009; 117: 1919-1924.

38 Jacquemin B, Sunyer J, Forsberg B, et al. Home outdoor $\mathrm{NO}_{2}$ and new onset of self-reported asthma in adults. Epidemiology 2009; 20: 119-126.

39 Modig L, Toren K, Janson C, et al. Vehicle exhaust outside the home and onset of asthma among adults. Eur Respir J 2009; 33: 1261-1267.

40 Zhu Y, Hinds W, Kim S, et al. Concentration and size distribution of ultrafine particles near a major highway. $J$ Air Waste Manag Assoc 2002; 52: 1032-1042.

41 Brugge D, Durant JL, Rioux C. Near-highway pollutants in motor vehicle exhaust: a review of epidemiologic evidence of cardiac and pulmonary health risks. Environ Health 2007; 6: 23-23.

42 Dadvand P, Ostro B, Figueras F, et al. Residential proximity to major roads and term low birth weight: the roles of air pollution, heat, noise, and road-adjacent trees. Epidemiology 2014; 25: 518-525.

43 HEI Panel on the Health Effects of Traffic-Related Air Pollution. Traffic-Related Air Pollution: A Critical Review of the Literature on Emissions, Exposure, and Health Effects. Special Report. Boston, Health Effects Institute, 2010 